\title{
Tratamiento anticoagulante y vasodilatador para el síndrome de Nicolau tras la administración de una inyección de bencilpenicilina benzatínica intramuscular a un niño de cuatro años de edad
}

\author{
Anticoagulant and vasodilator therapy for Nicolau syndrome following \\ intramuscular benzathine penicillin injection in a 4 year old boy
}

Dra. Tijen Alkan Bozkaya $a^{a}$ Dra. Gamze Demirel ${ }^{b}$, Dr. Tugrul Ormecic ${ }^{c}$ Dr. Serdar Al ${ }^{b}$, Dr. Engin Çakar ${ }^{d}$, Dr. Ayhan Tastekin ${ }^{b}$ y Dr. Halil Turkoglu

\section{RESUMEN}

El síndrome de Nicolau (SN) es una complicación rara de la inyección de ciertos fármacos por vía intramuscular, intrarticular o subcutánea, que produce necrosis isquémica de la piel, las partes blandas y el tejido muscular circundante. La bencilpenicilina benzatínica es uno de los antibióticos más ampliamente empleados para las infecciones de las vías respiratorias altas y raramente se ha notificado que produzca SN. En este artículo presentamos el caso de un niño de sexo masculino de cuatro años de edad diagnosticado con SN tras la inyección de bencilpenicilina benzatínica tratado satisfactoriamente con heparina fraccionada (enoxaparina) y pentoxifilina. Los médicos deben estar atentos al uso innecesario de bencilpenicilina benzatínica para evitar las probables complicaciones.

Palabras clave: síndrome de Nicolau, bencilpenicilina benzatínica, enoxaparina, pentoxifilina.

http:/ /dx.doi.org/10.5546/aap.2016.e184

\section{INTRODUCCIÓN}

El síndrome de Nicolau (SN), también conocido como embolia cutis medicamentosa o dermatitis livedoide, es una complicación rara de la inyección de ciertos fármacos por vía intramuscular,

a. Departamento de Cirugía Cardiovascular.

b. Departamento de Pediatría y Neonatología.

c. Departamento de Radiología.

d. Departamento de Medicina Física y Rehabilitación.

Medipol Üniversitesi, Estambul, Turquía.

\section{Correspondencia:}

Dra. Gamze Demirel: fgdemirel@medipol.edu.tr

Financiamiento: Ninguno.

Conflicto de intereses: Ninguno que declarar.

Recibido: 10-10-2015

Aceptado: 16-12-2015 intrarticular o subcutánea, que produce necrosis isquémica de la piel, las partes blandas y el tejido muscular. ${ }^{1,2}$ Se han notificado casos de SN con la administración de diversos fármacos, por ejemplo, antibióticos, anestesia local, corticoesteroides y antinflamatorios no esteroides. ${ }^{3,7}$

Aún no se ha dilucidado completamente la patogenia del SN. En el pasado, se atribuía a las inyecciones intrarteriales accidentales parte de su etiología. ${ }^{8}$ Recientemente, se han propuesto diferentes mecanismos, como el vasoespasmo agudo, la arteritis y la oclusión tromboembólica de las arterias pequeñas. ${ }^{9}$ Habitualmente se presenta como dolor alrededor del lugar de administración de la inyección, seguido de eritema, lesión livedoide y necrosis de las partes blandas. ${ }^{3,9,10}$ En este artículo presentamos el caso de un niño de sexo masculino de cuatro años de edad diagnosticado con $\mathrm{SN}$ tras la inyección de bencilpenicilina benzatínica intramuscular y tratado satisfactoriamente con terapia vasoactiva y antitrombótica.

\section{A PROPÓSITO DE UN CASO}

Derivaron a un niño de cuatro años de edad a nuestra sala de emergencias con dolor y edema en la extremidad inferior derecha. Los síntomas aparecieron pocas horas después de la inyección de una dosis de 600000 UI de bencilpenicilina benzatínica intramuscular para el tratamiento de una infección aguda de las vías respiratorias altas. Durante el examen físico se observó equimosis alrededor del lugar de administración de la inyección, y en pocos minutos aparecieron cambios livedoides, seguidos de un patrón isquémico (Figuras 1,2).

Unas pocas horas después, la erupción y la inflamación afectaron toda la pierna. El niño tenía dolor intenso e incapacidad motriz parcial en la región inferior de la pierna. En la 
ecografía se observó inflamación de las partes blandas alrededor del lugar de administración de la inyección y un aspecto heterogéneo difuso y edema en la grasa subcutánea y el tejido muscular (Figura 3). Los signos vitales estaban estables. En los parámetros de laboratorio no se observaron signos de infección ni de trastornos hematológicos. Al paciente se le diagnosticó SN. Se lo hospitalizó y se le administró heparina fraccionada (Clexane ${ }^{\circledR}$, por vía s.c.), dextrano (Rheomacrodex ${ }^{\circledR}$ ) y pentoxifilina $\left(\right.$ Trental $\left.{ }^{\circledR}\right)$. Esta terapia antitrombótica y vasodilatadora se inició dentro de la primera hora de hospitalización; la dosis de heparina fraccionada fue de $1,5 \mathrm{mg} / \mathrm{kg} /$ dosis, dos veces al día, durante ocho días, mientras que la dosis de pentoxifilina fue de $10 \mathrm{mg} / \mathrm{kg} /$ día durante cuatro días. Después del cuarto día de hospitalización, el paciente comenzó a caminar normalmente y recuperó su capacidad motriz por completo. En la ecografía de control se observó que el edema dentro de la grasa subcutánea y del tejido muscular había disminuido en comparación con la ecografía inicial.

\section{DISCUSIÓN}

El síndrome de Nicolau es una complicación rara e inexplicable de la inyección de diversos fármacos por vía intramuscular, intrarticular o subcutánea, caracterizado por necrosis aséptica de las partes blandas. ${ }^{11}$ Nicolau ${ }^{11}$ lo describió por primera vez en 1925, después de usar sales de bismuto por vía intramuscular para el

FiguRa 1. Durante el examen físico se observó erupción purpúrica alrededor del lugar de administración de la inyección, y en pocos minutos aparecieron cambios livedoides, seguidos de un patrón isquémico

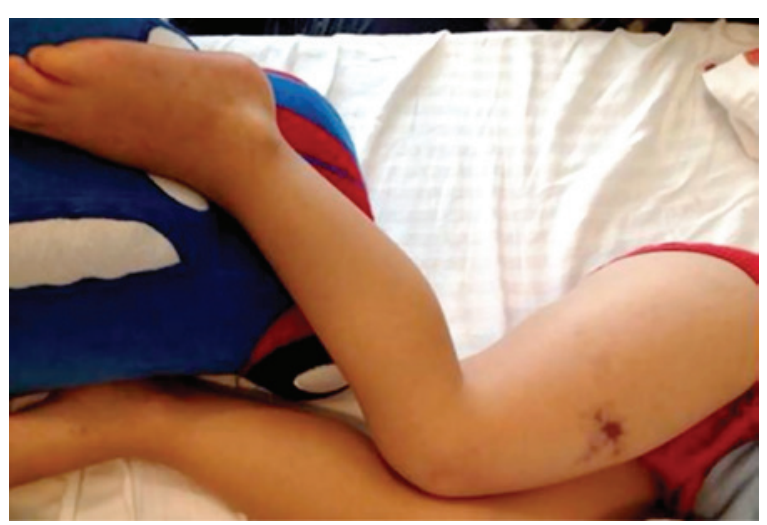

tratamiento de la sífilis. El SN se ha asociado a distintos fármacos, entre otros, antibióticos, corticoesteroides, antinflamatorios no esteroides, sedantes y vacunas. ${ }^{9,12}$ La penicilina fue uno de los primeros antibióticos asociados al SN. ${ }^{13-15}$ Se desconoce su patogenia, pero la hipótesis más plausible es la lesión vascular inicial, con inflamación local, espasmo reflejo de las arterias, trombosis arterial local y oclusión vascular por microémbolos. ${ }^{9}$ Lo más importante para la prevención del SN es el uso de una técnica adecuada al aplicar el medicamento y la aspiración antes de la inyección, y la aplicación de pequeñas cantidades (se sugiere menos de $5 \mathrm{ml}$ por lugar de administración).

El diagnóstico se basa en la presentación clínica, y la primera manifestación es un dolor local intenso inmediatamente después de inyectar el fármaco. Luego se observan palidez, lesiones cianóticas y un aspecto livedoide, que a veces provoca necrosis local. ${ }^{7}$ Nuestro paciente fue derivado a la sala de emergencias con dolor local intenso y equimosis en el lugar de administración de la inyección. Raramente, se produce deterioro neurológico en el SN. ${ }^{5}$ Nuestro paciente tenía incapacidad motriz parcial en la parte inferior de la pierna y no pudo caminar durante cuatro días después del episodio.

FiguRa 2. Unas pocas horas después de la erupción purpúrica alrededor del lugar de administración de la inyección, la erupción y la inflamación afectaron toda la pierna

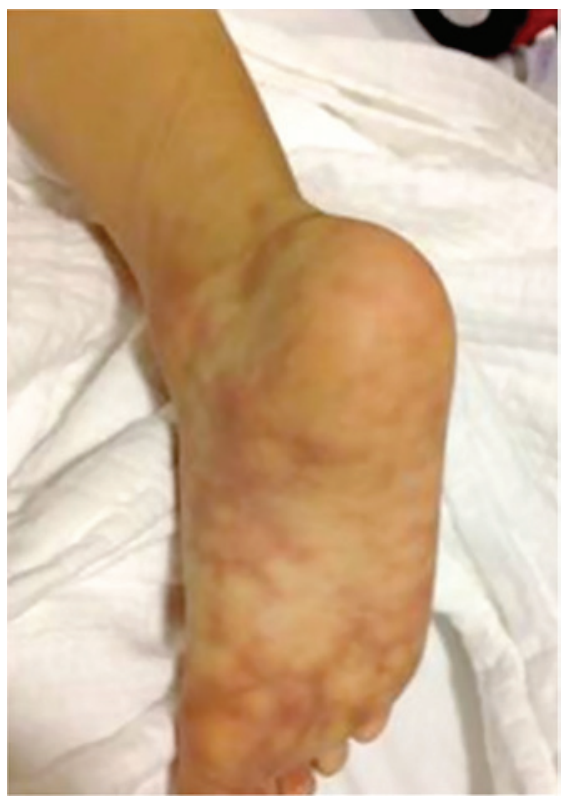


Figura 3. Imágenes ecográficas axiales. Aumento del grosor y la ecogenicidad de la grasa subcutánea (asterisco). A-También se observa el aumento de tamaño del músculo recto femoral derecho, que es el lugar de administración de la inyección. B-En la ecografía de seguimiento, se observa disminución del edema dentro la grasa subcutánea y reducción de tamaño de los músculos.

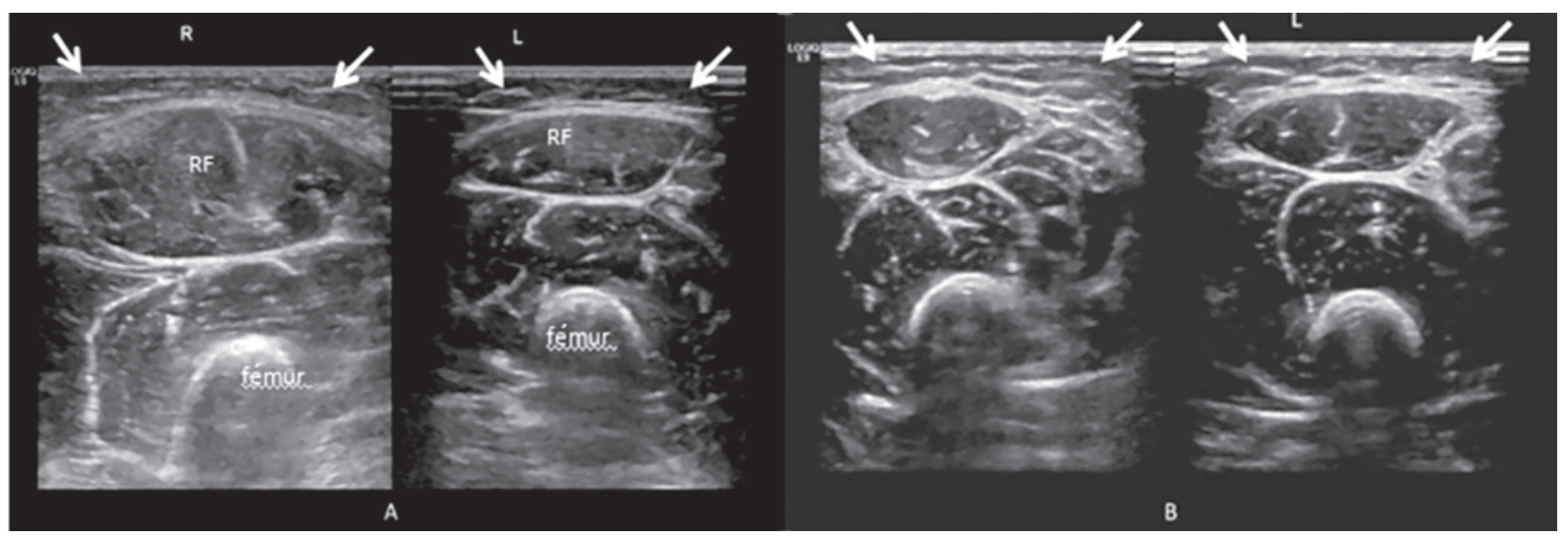

El tratamiento es sintomático e incluye manejo del dolor, profilaxis antibiótica e intervenciones quirúrgicas conservadoras. ${ }^{7}$ En las publicaciones científicas se menciona la eficacia de los vasodilatadores, los anticoagulantes y los esteroides sistémicos. ${ }^{10}$

Las publicaciones sobre el tratamiento anticoagulante son limitadas. En nuestro paciente se empleó heparina fraccionada (enoxaparina), dextrano y pentoxifilina, de manera muy satisfactoria. Se ha notificado que el oxígeno hiperbárico es eficaz cuando se producen lesiones por reperfusión. ${ }^{15} \mathrm{El}$ pronóstico es variable, y se trata de una afección potencialmente mortal con distintos niveles de necrosis tisular. La infección sistémica y la insuficiencia renal causada por la rabdomiólisis podrían provocar la muerte.

A modo de conclusión, los médicos deben estar atentos a las posibles complicaciones de las inyecciones intramusculares y deben evitar su aplicación innecesaria en niños. La anafilaxia es una de las complicaciones temidas de la inyección de penicilina, aunque las complicaciones de las inyecciones intramusculares no se limitan a estos problemas ampliamente reconocidos. Se debe considerar el SN frente a las manifestaciones clínicas específicas después de inyectar ciertos fármacos. La bencilpenicilina benzatínica es uno de los fármacos más indebidamente utilizados para las infecciones de las vías respiratorias altas y debería emplearse con mayor precaución para evitar las probables complicaciones.

\section{REFERENCIAS}

1. Kim SK, Kim TH, Lee KC. Nicolau syndrome after intramuscularinjection: 3 cases. Arch Plast Surg 2012;39(3):249-52.

2. Senel E. Nicolau syndrome as an avoidable complication. J Family Community Med 2012;19(1):52-3.

3. EzzedineK, Vadoud-SeyediJ,Heenen M. Nicolausyndrome following diclofenac administration. Br J Dermatol 2004; 150(2):385-7.

4. Senel E, Ada S, Gülec AT, Caglar B. Nicolau syndrome aggravated by cold application after i.m. diclofenac. J Dermatol 2008;35(1):18-20.

5. Lee DP, Bae GY, Lee MW, Choi JH, et al. Nicolau syndrome caused by piroxicam. Int J Dermatol 2005;44(12):1069-70.

6. Modzelewska I, Dawidowicz-Szczepanowska A. Nicolau syndrome following administration of procaine penicillin. Wiad Lek 1980;33(3):231-3.

7. Cherasse A, Kahn MF, Mistrih R, Maillard H, et al. Nicolau's syndrome after local glucocorticoid injection. Joint Bone Spine 2003;70(5):390-2.

8. Stiehl P, Weissbach G, Schröter K. Nicolau syndrome. Pathogenesis and clinical aspects of penicillin-induced arterial embolism. Schweiz Med Wochenschr 1971;101(11):377-85.

9. Luton K, Garcia C, Poletti E, Koester G. Nicolau Syndrome: three cases and review. Int J Dermatol 2006;45(11):1326-8.

10. Lie C, Leung F, Chow SP. Nicolau syndrome following intramuscular diclofenac administration: a case report. $J$ Orthop Surg (Hong Kong) 2006;14(1):104-7.

11. Nicolau S. Dermite livedoide et gangreneuse de la fesse, consecutive aux injections intra-muscularies dans la syphilis; a propos d'un cas d'embolic arteriellebismuthique. Ann Mal Vener 1925;20:321-9.

12. Cockshott WP, Thompson GT, Howlett LJ, Seeley ET. Intramuscular or intralipomatous injections? N Engl J Me 1982;307(6):356-8.

13. Noaparast M, Mirsharifi R, Elyasinia F, Parsaei R, et al. Nicolau syndrome after intramuscular benzathine penicillin injection. Iran J Med Sci 2014;39(6):577-9.

14. Benmiloud S, Hida M. Nicolau syndrome complicating an intramuscular injection of benzathine penicillin. Pan Afr Med J 2014;18:105.

15. Karimi M, Owlia MB. Nicolau syndrome following intramuscular penicillin injection. J Coll Physicians Surg Pak 2012;22(1):41-2. 